\section{Toward a Translationally Relevant Preclinical Model of Cannabis Use}

As the political and public landscape surrounding cannabis continues to shift, there is an urgent need to better understand how cannabis use impacts the brain and behavior. Preclinical studies are advantageous in this respect; however, there are drawbacks with current approaches that limit their translational value. Perhaps due to DEA-imposed restrictions, synthetic CB1R agonists have become the drug of choice in rodent models of cannabis exposure. However, the pharmacological properties of these compounds differ tremendously from that of cannabis, with synthetic CB1R agonists and THC recruiting different intracellular signaling pathways (Laprairie et al, 2014). Thus, the cellular effects of synthetic CB1R agonists often used in preclinical studies may not be indicative of the effects of THC, let alone cannabis. When THC is used, dosing is often excessively high and exposure regimens are not representative of human use patterns since a bolus THC injection differs dramatically from that of titrated cannabis inhalation. Moreover, cannabis is comprised of more than THC. There are over 120 phytocannabinoids in the cannabis plant, each with its own unique pharmacodynamic profile and the interaction between these phytocannabinoids may influence its effects. For instance, cannabidiol can inhibit THCdependent intracellular signaling (Laprairie et al, 2016) and attenuate THC-induced paranoia and memory deficits in humans (Englund et al, 2013). Therefore, THC alone may produce effects that are not indicative of human cannabis consumption. This is supported by human studies indicating that intravenous THC delivery produces a myriad of adverse events that are most often associated with higher doses and faster infusion rates (Carbuto et al, 2012). In this regard, it is not surprising that intravenous THC self-administration has been notoriously difficult to demonstrate in rodents. Finally, inhalation is the most common route of cannabis administration and the pharmacokinetics vary markedly depending on whether the drug is injected, inhaled, or injested. Additionally, whereas intraperitoneal THC elicits conditioned avoidance, intrapulmonary THC actually produces a place preference in rats (Manwell et al, 2014). Thus, in order to effectively model cannabis use and understand its effects on the brain and behavior, there must be a fundamental shift in the experimental approach. To this end, we are currently developing a novel vapor inhalation model that uses 'e-cigarette' technology to deliver discrete 'puffs' of vaporized cannabis extracts in a responsecontingent manner. This approach mimics the most common route of administration in humans while bypassing the aversive nature of forced drug exposure and avoiding the invasiveness of intravenous catheterization. Passive delivery of vaporized cannabis extracts has recently been validated (Nguyen et al, 2016), and our preliminary data indicate that cannabis vapor self-administration supports stable, physiologically relevant rates of drug delivery and produces robust conditioned drug-seeking behavior. Establishing this more translationally relevant model will afford empirical evaluation of mainstream beliefs regarding the effects of cannabis (eg, more deleterious effects following early-onset cannabis use or the use of high THC preparations), permit finer interrogation of the effects of cannabis on the brain, and help to identify genetic or environmental factors that may increase vulnerability for developing cannabis-related problems.

\section{FUNDING AND DISCLOSURE}

This research was supported by a pilot grant from the Washington State University Alcohol and Drug Abuse
Research Program and an intramural grant from the Washington State University College of Veterinary Medicine. The author declares no conflict of interest.

\section{Ryan J McLaughlin ${ }^{1,2,3}$ \\ ${ }^{1}$ Department of Integrative Physiology \& Neuroscience, Washington State University, Pullman, WA, USA; ${ }^{2}$ Department of Psychology, Washington State University, Pullman, WA, USA; ${ }^{3}$ Translational Addiction Research Center, Washington State University, Pullman, WA, USA \\ E-mail: ryanmclaughlin@vetmed.wsu.edu}

Carbuto M, Sewell RA, Williams A, Forselius-Bielen K, Braley G, Elander J et al (2012). The safety of studies with intravenous $\Delta^{\prime}$-tetrahydrocannabinol in humans, with case histories. Psychopharmacology (Berl) 219: 885-896.

Englund A, Morrison PD, Nottage J, Hague D, Kane F, Bonaccorso S et al (2013). Cannabidiol inhibits THC-elicited paranoid symptoms and hippocampal-dependent memory impairment. J Psychopharmacol 27: 19-27.

Laprairie RB, Bagher AM, Kelly ME, Denovan-Wright EM (2016). Biased type 1 cannabinoid receptor signaling influences neuronal viability in a cell culture model of Huntington disease. Mol Pharmacol 89: 364-375.

Laprairie RB, Bagher AM, Kelly ME, Dupré DJ, Denovan-Wright EM (2014). Type 1 cannabinoid receptor ligands display functional selectivity in a cell culture model of striatal medium spiny projection neurons. J Biol Chem 289: 24845-24862.

Manwell LA, Charchoglyan A, Brewer D, Matthews BA, Heipel H, Mallet PE (2014). A vapourized $\Delta(9)$ tetrahydrocannabinol $(\Delta(9)$-THC) delivery system part I: development and validation of a pulmonary cannabinoid route of exposure for experimental pharmacology studies in rodents. I Pharmacol Toxicol Methods 70: 120-127.

Nguyen JD, Aarde SM, Vandewater SA, Grant Y, Stouffer DG, Parsons LH et al (2016). Inhaled delivery of $\Delta(9)$-tetrahydrocannabinol (THC) to rats by e-cigarette vapor technology. Neuropharmacology 109: 112-120.

Neuropsychopharmacology Reviews (2018) 43, 213. doi: 10.1038/npp.2017.19|

\section{Setting the Legal Age for Access to Cannabis in Canada: Bridging Neuroscience, Policy, and Prevention}

The United States and Canada are both engaged in cannabis policy reforms with a number of US states legalizing 